\title{
Realization of Interworking in LTE Roaming Using a Diameter-based Interworking Function
}

\author{
Arnab Dey, Balaji Rajappa and Lakshman Bana \\ Engineering, Diametriq, Melbourne, USA; \\ e-mail: $\{$ adey, brajappa,lbana $\} @$ diametriq.com
}

Received 15 January 2013; Accepted 17 February 2013

\begin{abstract}
While operators around the world are onboarding or planning to onboard the LTE-based Evolved Packet System (EPS), some troubling questions linger on. The prospects of LTE are enticing. However, would the LTE and Legacy networks interwork and co-exist? Would inbound/outbound roamers face any service disruption? For a green-field operator, possibly with a tight budget, is adopting LTE a risky proposition when over $90 \%$ of the world's network is still based on Legacy Signaling System No. 7 (SS7)? Various standards bodies have provided guidelines and specifications to identify and address some of the interworking and co-existence scenarios, but their implementation is complex and requires a detailed knowledge of the disparate worlds of EPS and Legacy protocols in finding an acceptable intersection between the two.

This paper describes the important interworking issues between Diameter and TCAP (SS7)-based protocols and some of the practical aspects that transcend information that is disseminated through the standards bodies. Some specific scenarios such as a $2 \mathrm{G} / 3 \mathrm{G}$ subscriber roaming into an LTE network using home-routed applications and S8 (EPS) - Gp (Legacy) interworking are not covered here and shall be discussed in a subsequent paper. Similarly, this paper has not focused on Diameter-RADIUS interworking needed to support RADIUS-based AAA Server. However, most other scenarios have
\end{abstract}

Journal of Cyber Security and Mobility, Vol. 1, 289-308.

(C) 2013 Diametriq, LLC. All rights reserved. 
been addressed and it is hoped that the solution presented here will alleviate the roaming, co-existence and interworking concerns of the reader.

Keywords: IWF, interworking, diameter, TCAP, LTE, MAP, CAP.

\section{Abbreviations}

AAA Authentication Authorization Accounting

ACN Application Context Name

ASN.1 Abstract Syntax Notation 1

AVP Attribute Value Pair

CAP CAMEL Application Part

EIR Equipment Identity Register

EPS Evolved Packet System

E-UTRAN Evolved UTRAN

GERAN GPRS Edge RAN

GPRS General Packet Radio Service

HLR Home Location Register

HPLMN Home PLMN

IE Information Element

IMSI International Mobile Subscriber Identity

IWF Inter Working Function

LTE Long Term Evolution

MAP Mobile Application Part

MCC Mobile Country Code

MNC Mobile National Code

MSIN Mobile Subscriber Identification Number

OCS Online Charging System

PCC Policy and Charging Control

PLMN Public Land Mobile Network

QoE Quality of Experience

RAN Radio Access Network

RADIUS Remote Authentication Dial-In User Svc

SCP Service Control Point

SCTP Stream Control Transmission Protocol

SIGTRAN Signaling Transport

SS7 $\quad$ Signaling System No. 7

TBCD Telephony Binary Coded Decimal

TCAP Transaction Capabilities Application Part 


$\begin{array}{ll}\text { TCP } & \text { Transmission Control Protocol } \\ \text { UE } & \text { User Equipment } \\ \text { UTRAN } & \text { Universal Terrestrial RAN } \\ \text { VPLMN } & \text { Visited PLMN }\end{array}$

\section{Introduction}

\subsection{Understanding of Common Terms}

The terms LTE, E-UTRAN, EPC and EPS have been used at various places in the text. It is important to have a lucid understanding of these terms and how they relate to each other before proceeding further.

The terms LTE and E-UTRAN are commonly used interchangeably. EUTRAN actually refers to the RAN that uses LTE, which is the radio interface technology.

EPC is the core network that uses E-UTRAN.

EPS comprises the UE, E-UTRAN, EPC and other access networks connecting through the EPC.

LTE also being the name of the 3GPP work item that developed EUTRAN and the corresponding radio interface technology is normally used in a broader scope in everyday usage.

\subsection{The Lure of LTE}

The LTE radio network provides an enriched Quality of (User) Experience (QoE) through higher peak data rates, lower latency and higher spectral efficiency. Thus, it can support the needs of today's bandwidth hogging Internet applications. The EPS architecture is designed to interwork $2 \mathrm{G} / 3 \mathrm{G}$, trusted non-3GPP (such as CDMA) and non-trusted non-3GPP (such as WLAN) accesses. The centralized Policy Control and Charging (PCC) framework in EPS allows Subscriber and Service differentiation, at the same time paving the way for optimized user and network resource usage.

\subsection{The Dilemma with LTE}

Had EPS been a monolithic architecture comprising only LTE, E-UTRAN and EPC, the promise of EPS' "advanced" technology would have been more palatable to the user community. However, EPS is supposed to interwork with heterogeneous networks, most of which have been there for a long time. Each 


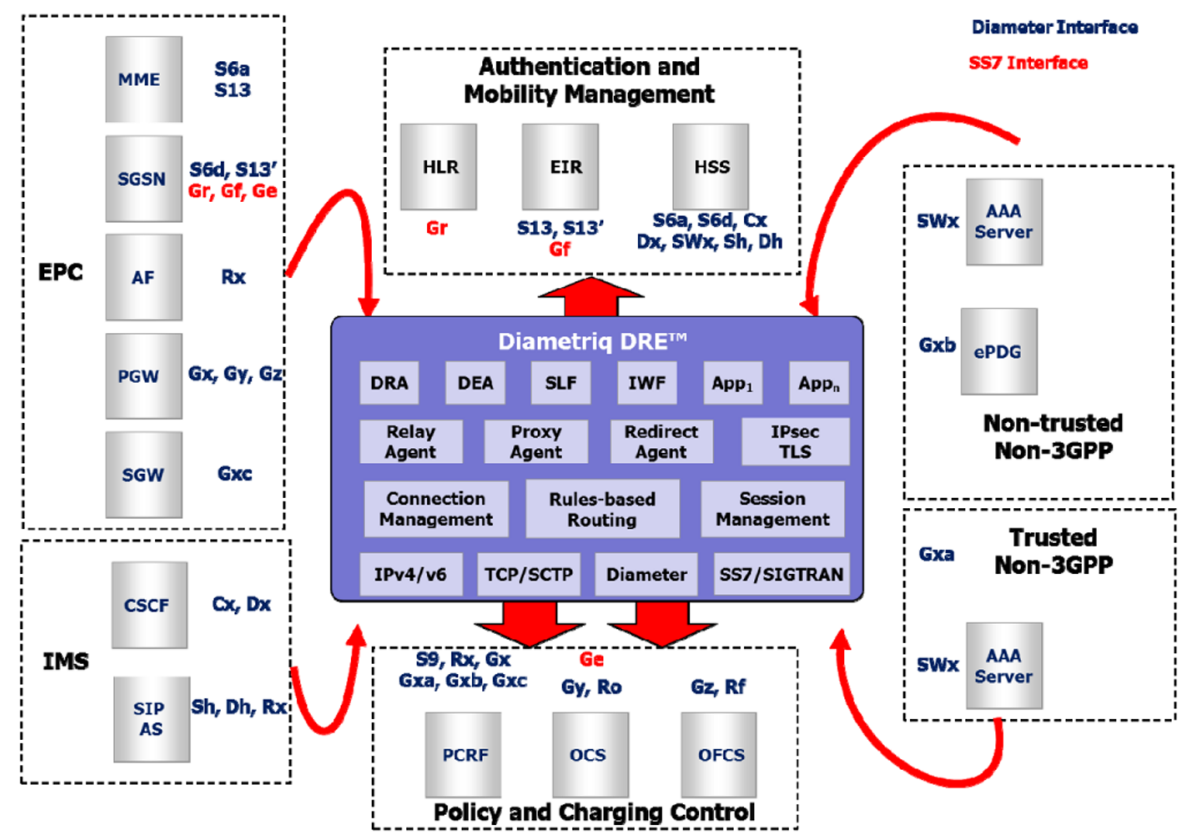

Figure 1 Diameter \& SS7 interfaces in the EPS ecosystem.

of these networks has its unique technology foundation. Integrating the varied technologies into the single EPS umbrella is a daunting task.

\subsection{Towards a Solution}

Appreciating the magnitude of the task, standards bodies have started specifying the various and various kinds of interworking scenarios. The approach has been to first identify and address those scenarios that roamers are more likely to encounter.

"Diameter" is the protocol for Authentication, Authorization and Accounting (AAA) in the EPS. It is used for Authentication, Mobility Management, Policy and Charging Control procedures. The Diameter interfaces supporting these procedures are illustrated in Figure 1. Furthermore, Table 1 illustrates the various SS7 interfaces and the corresponding serving network entities.

This paper focuses on the interaction of the Gr, Gf and Ge SS7 and related Diameter interfaces in the following scenarios: 
Table 1 Mapping of SS7 interfaces into serving network entities.

\begin{tabular}{ll}
\hline SS7 Interface & Network Entity \\
\hline $\mathrm{Gr}$ & HLR \\
$\mathrm{Gf}$ & EIR \\
$\mathrm{Ge}$ & OCS (Prepaid SCP) \\
\hline
\end{tabular}

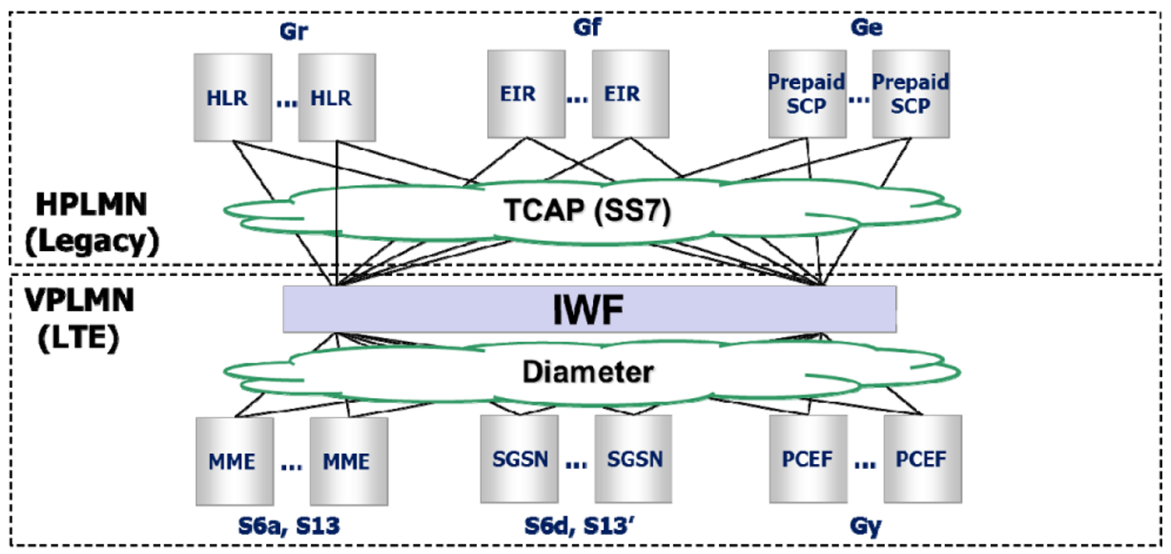

Figure 2 HPLMN is Legacy, VPLMN is LTE.

- HPLMN is Legacy, VPLMN is LTE - A 2G/3G subscriber roaming into an E-UTRAN.

- HPLMN is LTE, VPLMN is Legacy - A 4G subscriber roaming into an GERAN/UTRAN.

The above roaming scenarios are pictorially depicted in Figures 2 and 3. The InterWorking Function (IWF) mediates between two disparate protocols and makes them interoperable. It is assumed that the IWF is located in the EPS.

Table 2 Diameter - TCAP interworking in various roaming scenarios.

\begin{tabular}{lllll}
\hline \multicolumn{2}{l}{ HPLMN is Legacy, VPLMN is LTE } & & \multicolumn{2}{l}{ HPLMN is LTE, VPLMN is Legacy } \\
\cline { 1 - 2 } Diameter & TCAP & & TCAP & Diameter \\
\hline S6a & Gr (MAP) & & Gr (MAP) & S6d \\
S6d & Gr (MAP) & & Gf (MAP) & S13' \\
S13 & Gf (MAP) & & Ge (CAP) & Gy \\
S13 & Gf (MAP) & & \\
Gy & Ge (CAP) & & \\
\hline
\end{tabular}




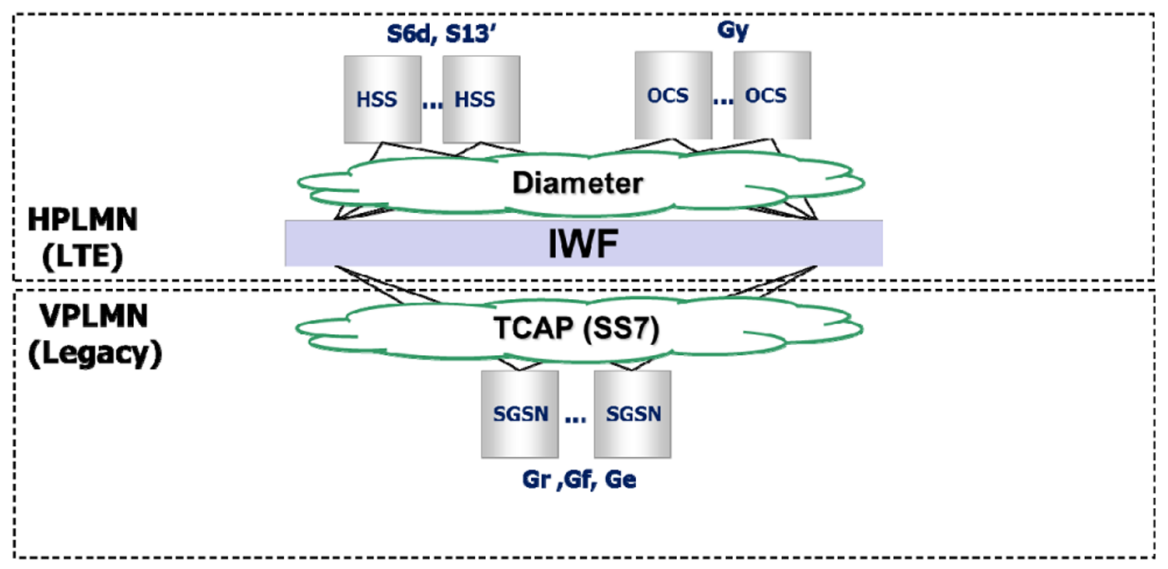

Figure 3 VPLMN is Legacy, HPLMN is LTE.

\section{The Solution}

The upcoming subsections unravel the nuts and bolts of the IWF.

\subsection{IWF Stack Diagram}

The IWF comprises a layered architecture having the following layers:

- Protocol - Every transaction in an interworking scenario involves two underlying protocols, for example, Diameter and TCAP. These operate independent of each other and comply with their "separate" protocol specifications as far as parameter and message encoding and decoding, message routing and protocol management is concerned. Supporting a new interworking entails supporting a new protocol.

- Transport Handler - This abstracts the usage of the corresponding underlying transport from the higher layers. Thus, there is one Transport Handler relevant to each protocol. Diameter is supported over TCP/SCTP. TCAP is supported over SIGTRAN/SS7.

- Transaction Manager - This is a singleton that manages the interworking transactions/sessions. It uses a State Machine in determining how to deal with an incoming request or response, and whether protocol conversion should be invoked. The beauty of the State Machine is its generality, which lends easy extensibility to the IWF in supporting a newer interworking. 


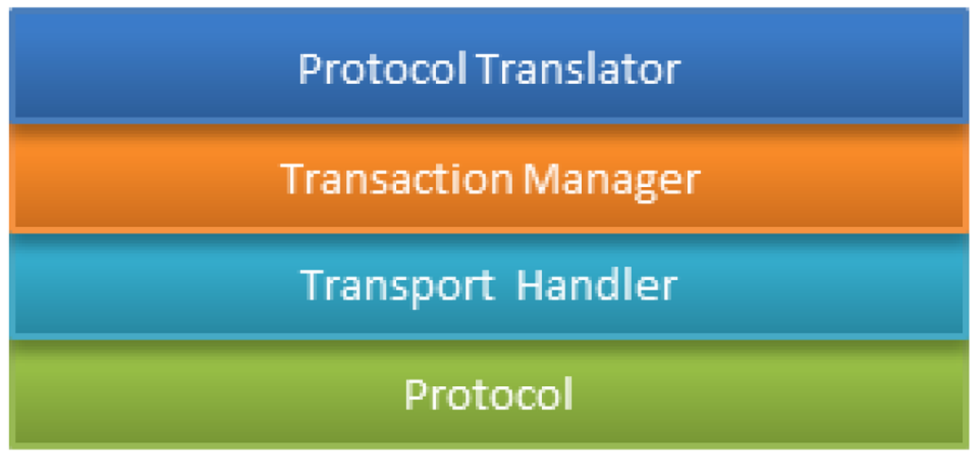

Figure 4 IWF state diagram.

- Protocol Translator - This is what performs the parameter and message mapping from one protocol to the other. Some of the protocol translations have been defined by standards bodies, for example, that between Diameter and MAP based interfaces [1]. Some others, for example, that between Diameter and CAP, have not yet been defined by any standards body - such protocol translations can be defined through knowledge of the corresponding protocols and interworking scenarios.

The IWF layered architecture has been depicted in Figure 4.

\subsection{Transport Handling}

Transport handling depends on the idiosyncrasies of a particular protocol. While a discussion of a protocol involved in an interworking and its transport related aspects is outside the scope of this paper, it is worthwhile to represent the two protocol suites used as baseline in this paper (Diameter \& TCAP). This helps us get a peek into the disparate nature of the two protocols and the ensuing complexity of interworking - an interested but uninitiated reader is encouraged to study the relevant protocol specifications to appreciate this even better.

\subsection{Transaction Manager}

This is the control center of the IWF. It is invoked when a protocol message arrives. At the time of invocation it knows the protocol of the received message. It then determines the following:

1. Whether the message is a request or response. 


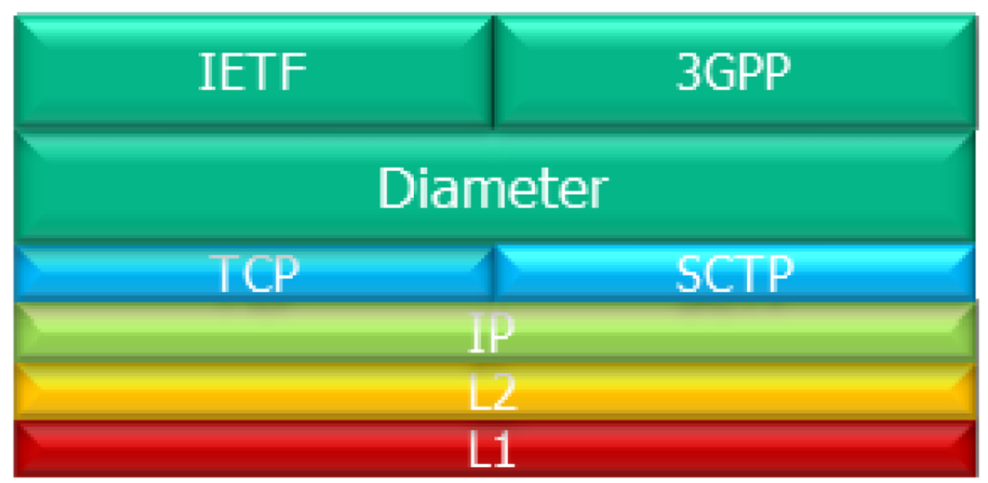

Figure 5 Diameter protocol suite.

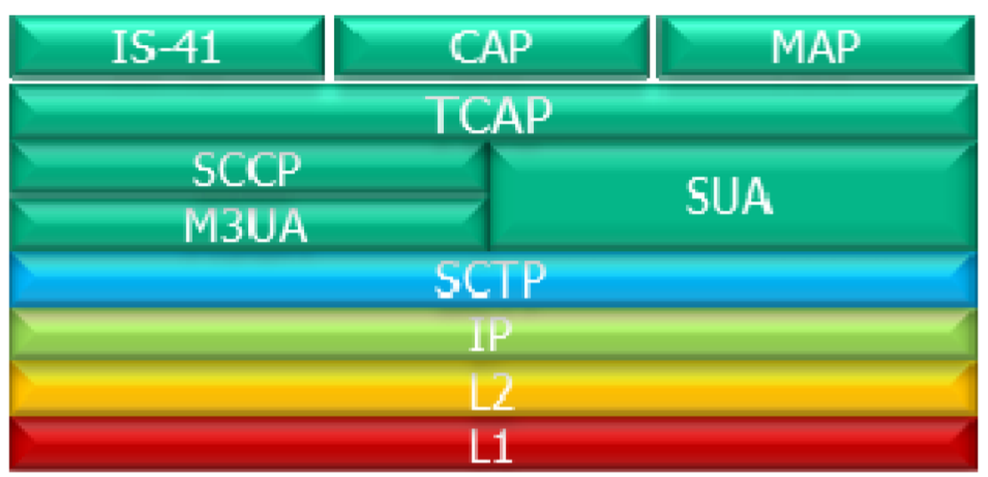

Figure 6 SIGTRAN protocol suite.

2. In the case of the TCAP protocol, whether the message is a TCAP dialog or component and what is the dialog or component type. This is because the TCAP protocol is complex in nature. A TCAP request consists of an optional dialog portion and one or more components. Some TCAP primitives are only generated and sent "locally" from the TCAP stack to the Transaction Manager, for example, when a response has not been received before a timer expiry. Refer to [3] for a functional description of the transaction capabilities of TCAP.

3. In the case of the TCAP protocol, the TCAP transaction the TCAP dialog or component belongs to.

4. If it is the "first" message of a transaction, the protocol at request origination. For example, when a $2 \mathrm{G} / 3 \mathrm{G}$ subscriber roams into an LTE 
area and switches on his UE, the ensuing authentication and mobility management procedures cause Diameter S6a/S6d transactions to be initiated at the MME/SGSN towards the HLR (3GPP Release 8 or later). In this case, the protocol at request origination is Diameter. Similarly, when in another scenario, an LTE subscriber roams into a $2 \mathrm{G} / 3 \mathrm{G}$ area and switches on his UE, the ensuing authentication and mobility management procedures cause MAP Gr transactions to be initiated at the SGSN (3GPP Release 8 or later). In this case, the protocol at request origination is MAP.

Based on the message received, the Transaction Manager determines the unique context the message belongs to. In these cases, "all" protocol messages belonging to the "same" transaction contain the "same unique" transaction identifier. In Diameter, this transaction identifier is referred as session identifier (refer to "Session-Id" in [2]). In TCAP, each of the two TCAP enduser applications, for example, the TCAP end-user applications residing in IWF and HLR respectively, is called a TC-user ("TC" refers to Transaction Capabilities). In each transaction in TCAP, each TC-user maintains its own transaction identifier. The origination and destination transaction identifiers are swapped depending on the direction of a message in the same transaction. Unique "dialogue identifier" maps onto the transaction identifiers exchanged in messages belonging to the same transaction in either direction (refer to "Dialogue ID" in [3]). The Transaction Manager maintains the following two mappings for an IWF transaction:

- In the case of a message received on Diameter, a mapping of the SessionId to a unique IWF context.

- In case of a message received on TCAP, a mapping of the Dialogue ID to the same IWF context.

As a result of the determination of (1), (2), (3), and (4), the Transaction Manager is able to compute the IWF Event that has occurred on a transaction. In case of the "first" message of a new transaction it creates a new IWF context and establishes a mapping of the newly created context with the received Diameter Session-Id or TCAP Dialogue ID; else it derives the stored IWF context based on the received Session-Id or Dialogue ID.

On the IWF context, the Transaction Manager then invokes the IWF State Machine, passing it the event and protocol message received. The State Machine has been developed keeping the various interworking scenarios in mind. It is designed to support "any" interworking, remaining unaware of the underlying protocols themselves. Thus the framework can be easily extended 


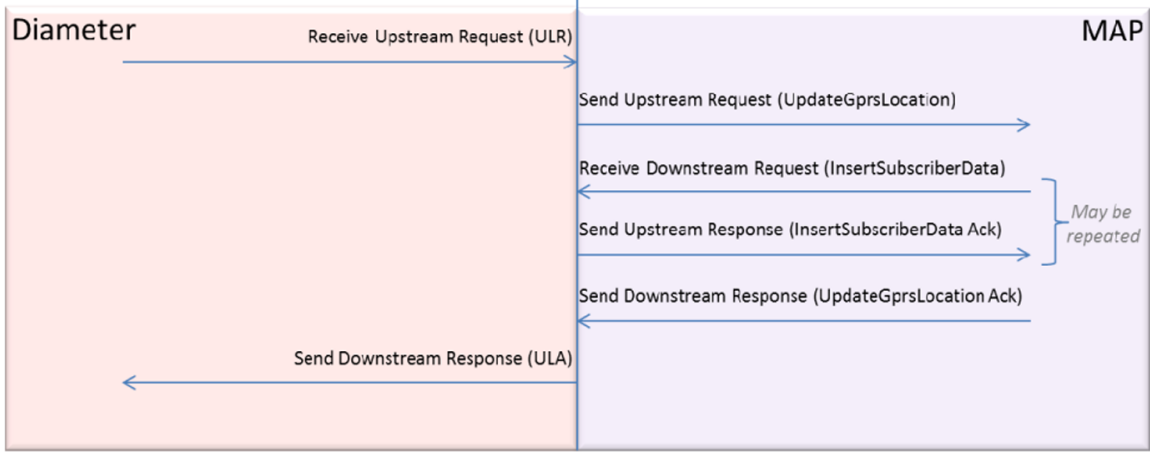

Figure 7 "With" interim request-response on MAP.

to support an additional interworking, for example, Diameter-RADIUS. The State Machine maps a possible incoming event and current state of the IWF context to a succeeding state and corresponding actions.

Figures 7, 8, 9 and 10 depict four examples, which among them represent "all" interworking scenarios for Diameter-MAP shown in Figures 2 and 3. These figures illustrate the generality of the State Machine.

In these figures, "upstream" refers to a direction from the Originating/IWF to the Terminating entity, with respect to a request or response. In Diameter-MAP interworking, the Originating entity is MME/SGSN and Terminating entity is HLR; an upstream request originates from MME/SGSN to IWF, or IWF to HLR; an upstream response originates from IWF to HLR. Correspondingly, "downstream" refers to a direction from the Terminating/IWF to the Originating entity, with respect to a request or response. In Diameter-MAP interworking, a downstream request originates from HLR to IWF, or IWF to MME/SGSN; a downstream response originates from HLR to IWF, or IWF to MME/SGSN.

\subsection{Protocol Translator}

This is the knowledge engine of the IWF. It is invoked when the Transaction Manager determines a protocol conversion is required. Implementing protocol translation between disparate protocols requires knowledge and ex- 


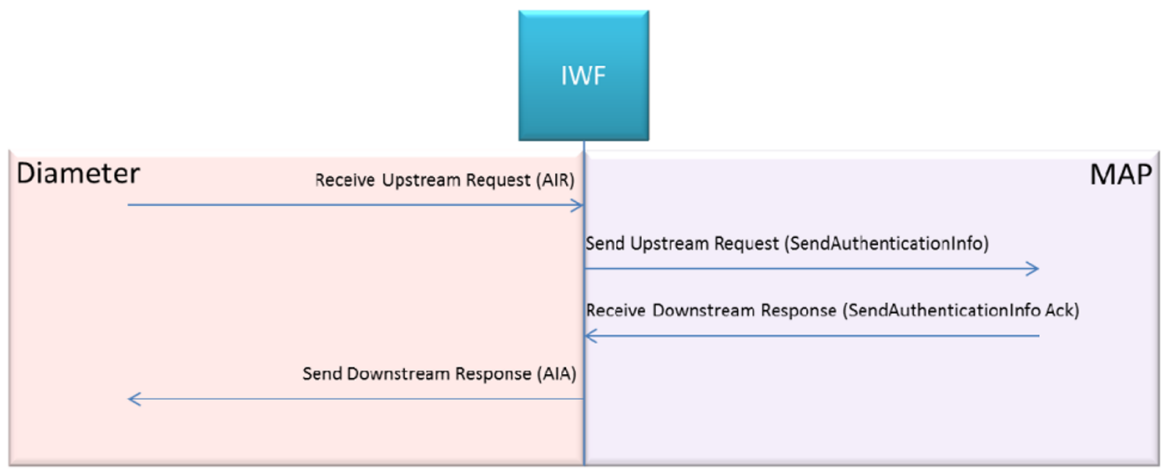

Figure 8 With "no" interim request-response on MAP.

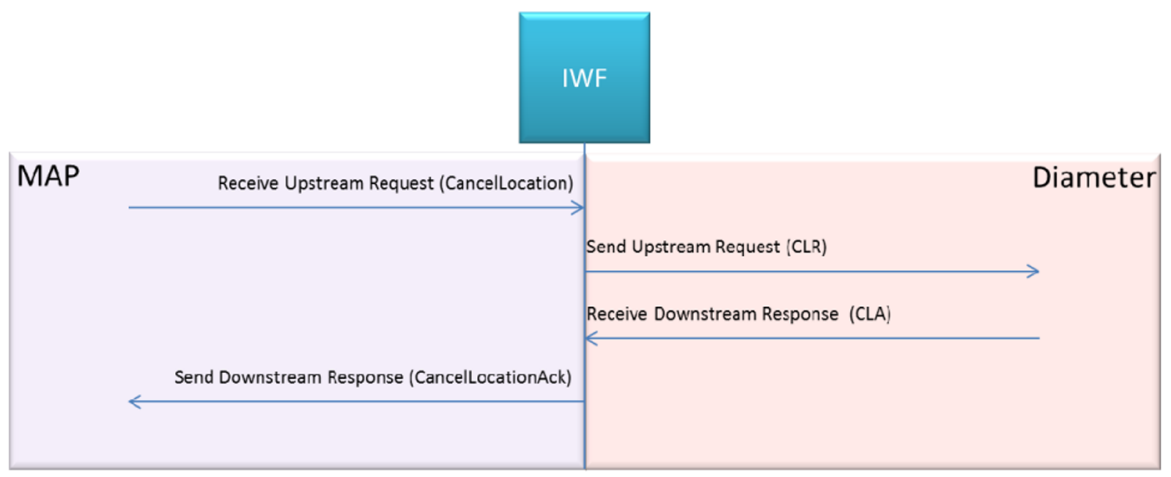

Figure 9 Request originated from MAP (including server-initiated).

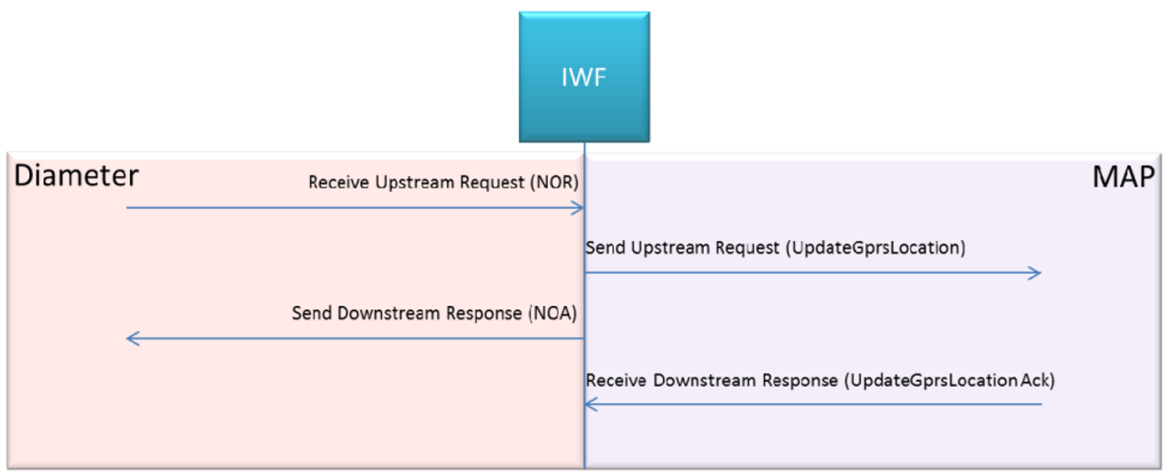

Figure 10 Diameter response sent without waiting for a MAP response. 
perience in the encoding and decoding of parameters and an understanding of the correlation between corresponding parameters of the respective protocols.

In the case of Diameter, a parameter is encoded in an Attribute Value Pair (AVP). The concept of AVP and the various AVP types are specified in [2]. Composition of the Diameter S6a/S6d messages, connotation of the AVPs an S6a/S6d message contains and description of their usage can be found in [8].

In the case of MAP/CAP over TCAP, a parameter is encoded in an Information Element (IE). Each protocol (MAP/CAP) request, response or error encoding is specified through the corresponding ASN.1 specifications, for example, [7] for MAP (Gr).

When it comes to interworking two protocols such as Diameter and MAP, the mapping of corresponding parameter is described in a home-grown specification or that from a standards organization. For example, the mapping of Diameter-MAP in Figure 2 is specified in [1]. How to implement the mapping requires algorithmic and programmatic skills.

In Table 2, AVP refers to a field in Diameter and IE refers to a field in MAP (or CAP). For a description of the various field names and types cited in Table 3, the reader can refer to [1, 2, 6-8].

\subsection{Approaches to Building a Protocol Translator}

There are two alternative approaches to building a Protocol Translator:

1. Logic coded in a high-level programming language such as $\mathrm{C} / \mathrm{C}++$.

2. Logic coded in a certain grammar-based scripting language.

Factors determining the chosen approach:

a. Availability of skilled personnel in the language of choice

b. Whether a grammar-based scripting language is already available, or has to be created

c. Time-to-market needs

If a grammar-based scripting language is the chosen approach, Table 3 illustrates the capabilities that the scripting language must support. Thus, the scripting language has to be tailored to the need. It might be possible to incorporate new parameter mappings on the fly. It might also make it easier to code parameters mappings. However, the grammar and scripting language has to be first defined to derive the benefits out of this approach. 
Table 3 Generic translation requirements for the IWF.

\begin{tabular}{|c|c|c|}
\hline Sl. No. & Requirement & Description \\
\hline \multirow[t]{5}{*}{1} & $\begin{array}{l}\text { Translation from/to an AVP } \\
\text { to/from an IE }\end{array}$ & $\begin{array}{l}\text { This is a straightforward translation from/to an } \\
\text { AVP to/from an IE, which may involve one of } \\
\text { the following types: }\end{array}$ \\
\hline & & $\begin{array}{l}\text { 1. Enumerated to Enumerated } \\
\text { 2. Bit string to bit string } \\
\text { 3. TBCD string to TBCD string }\end{array}$ \\
\hline & & Examples: \\
\hline & & $\begin{array}{l}\text { 1. RAT-Type AVP to usedRAT-Type IE } \\
\text { (Enumerated to Enumerated) } \\
\text { 2. Supported-Features. Feature-List AVP to } \\
\text { supportedFeatures IE (Bit string to bit } \\
\text { string) (Feature-List is Unsigned32) }\end{array}$ \\
\hline & & $\begin{array}{l}\text { 3. MME-Number-for-MT-SMS AVP to } \\
\text { mmeNumberforMTSMS IE } \\
\text { string to TBCD string) }\end{array}$ \\
\hline 2 & $\begin{array}{l}\text { Extraction of a part of an AVP } \\
\text { to populate an IE }\end{array}$ & $\begin{array}{l}\text { In this, a part of an AVP is extracted and the } \\
\text { resulting value is put into an IE. Typically, such } \\
\text { an extraction can be performed using string } \\
\text { manipulation. } \\
\text { Example: } \\
\text { User-Name AVP to imsi IE }\end{array}$ \\
\hline 3 & $\begin{array}{l}\text { Generation of a parameter using } \\
\text { another AVP }\end{array}$ & $\begin{array}{l}\text { In some cases, an IE cannot be directly pop- } \\
\text { ulated from any AVP. But, possible values for } \\
\text { a certain AVP can be used to define a static } \\
\text { mapping, locally. At runtime, a lookup is per- } \\
\text { formed for the received AVP value, the mapped } \\
\text { value obtained from the configuration, and the } \\
\text { IE populated using the mapped value. } \\
\text { Example: } \\
\text { sgsn-Address IE obtained through the Origin- } \\
\text { Host AVP }\end{array}$ \\
\hline 4 & $\begin{array}{l}\text { Setting an IE or AVP to present } \\
\text { (IE), or constant value }\end{array}$ & $\begin{array}{l}\text { In some cases, for an IE or AVP, there is no cor- } \\
\text { responding AVP or IE. In such a case, the IE or } \\
\text { AVP is populated with a constant value (may be } \\
\text { defined through configuration). Further, in case } \\
\text { of MAP, an IE can just be encoded as present in } \\
\text { the outgoing MAP message. } \\
\text { Example: } \\
\text { gprsEnhancementsSupportIndicator IE is al- } \\
\text { ways encoded to be present - in this case, there } \\
\text { is no corresponding incoming AVP. }\end{array}$ \\
\hline
\end{tabular}


Table 3 (Continued)

5 Absence of an IE or AVP In some cases, an IE or AVP is absent, i.e., is

not encoded in the message.

Example:

extensionContainer parameter.

6 Translation of an enumerated

In some cases, an enumerated type is used to type to bit string populate a bit string.

Example:

RAT-Type AVP to supportedRAT-

TypesIndicator IE.

7 Custom function to combine In some cases, two or more AVPs or IEs need two or more AVPs or IEs into a single IE or AVP to be combined into a single IE or AVP. In such cases, a custom function is used.

Example:

Terminal-Information.IMEI (UTF8String $\rightarrow$

14-digit) and Terminal-Information.SoftwareVersion (UTF8String $\rightarrow$ 2-digit) AVP values is combined and encoded into add-info.imeisv IE as an OCTET STRING (ASN.1).

8 Encoding presence of an IE In some cases, an IE is present if a certain bit is based on a certain bit set in an AVP

set in an AVP.

Example:

servingNodeTypeIndicator IE is present if the ULR-Flags AVP has the S6a/S6d Indicator bit set

9 Encoding presence of an IE In some cases, an IE is present if a certain bit is based on a certain bit NOT set in an AVP

NOT set in an AVP.

Example:

gprsSubscriptionDataNotNeeded IE is present if the ULR-Flags AVP has the GPRS Subscription Data Indicator bit NOT set

10 Encoding presence of an IE In some cases, an IE is present based on the based on a binary Enumerated AVP enumerated value of an AVP. The enumerated value can be either 0 or 1 . That is, if the value is 0 , the IE is not present; if the value is 1 , the IE is present. Default behavior is the absence of the IE (if the AVP is NOT present).

Example: 
Table 3 (Continued)

11 Translation of an IE based on a A condition checks the presence or the absence condition applied on the incoming message value is set to the AVP.

\section{Example:}

In the translation of UpdateGPRSLocationRes/Err to ULA, the Result-Code AVP is mapped to an error code based on the presence or absence of a particular IE.

12 Possible cases:

In the case of TCAP based applications, there

- All the translations are specified at the transaction level.

- There are events within a transaction to allow error components and intermediate message components.

- There are IE or AVP mappings/settings within events

- The translation has a provision to allow state variables, which are valid for the entire transaction state, to store intermediate results in AVPs, IEs or other basic types

- The translation allows the setting of state variables to IEs or AVPs within an event

- The translation allows set operations from AVPs or IEs to a state variable

- The translation allows IE mappings/settings within a transaction or an event can be multiple messages exchanged within a transaction. But all might map to a single answer on the Diameter side.

Example:

In the translation of UpdateGPRSLocationRes/Err to ULA, if the InsertSubscriberDataArg was sent as part of the same transaction and if the SkipSubscriber-Data is not present in the previously sent ULR, then the Subscription-Data AVP is populated from the InsertSubscriberDataArg. But the ULA is sent only when the UpdateGPRSLocationRes/Err is received. 
Table 3 (Continued)

13 Translation allows the mapping In the ASN.1 notation, an IE that repeats mulof an IE of type SEQUENCE to tiple times is denoted as a SEQUENCE. Diaa Grouped AVP and vice-versa meter has the notion of Grouped AVP. The translation allows such a mapping possible. Example: In the translation of InsertSubcriberDataArg to Subscription-Data AVP, RegionalSubscription- Zone-Code is of type OctetString (AVP type) but has a maximum multiplicity of 10. InsertSubcriberDataArg .subscriberData.regionalsubscriptionData, which is a Sequence of Octet String (ASN.1) maps to this.

14 Translation allows the following conversions possible

- DNS encoded to UTF8String (displayable string) and vice versa

- OCTET STRING (ASN.1) to their Hexadecimal display string equivalent and vice versa

- TBCD encoded string to UTF8String (displayable string) and vice versa
15 Translation allows mapping from a SEQUENCE IE to a Grouped AVP and vice versa
DNS encoding of APN, which is nothing but an FQDN, is different from the string representation of a domain name. Diameter uses the plain string representation of the domain name, but this is not the case with legacy protocols. Hence, this is supported. Similar conversions are required for other parameters.

Examples:

- APN-OI-Replacement from apn-oireplacement in InsertSubscriberDataArg

- 3GPP-Charging-Characteristics is a displayable string (4 bytes), while the charging Characteristics of InsertSubsDataArg is an OCTET STRING (ASN.1) of 2 bytes

- IMSI is encoded as UTF8String in Diameter while that is not the case with legacy protocols

Nesting of IEs is possible in ASN.1 with the SEQUENCE type. Similarly, nesting of AVPs is possible in Diameter with the Grouped AVP type. There is a construct that maps the nested IEs to AVPs within a Grouped AVP. This is allowed at multiple levels of nesting.

Example:

In the translation of InsertSubscriberDataArg to ULA.Subscription-Data, SubscriptionData.AMBR Grouped AVP (with two sub-AVPs) is mapped to ISDArg.epsSubscriptionData.ambr which is a SEQUENCE type with two parameters 
Table 3 (Continued)

16 Translation allows the mapping of a SEQUENCE(nesting) of SEQUENCE (list) to repeated Grouped AVP and vice versa

17 Translation allows the following conversions

- IE presence to single valued Enumerated AVP

- Boolean IE to binary valued Enumerated AVP

- IE presence to binary valued Enumerated AVP
In the ASN.1 notation, a SEQUENCE can denote grouping of IEs and also denote a list/array. Similarly, in Diameter, Grouped AVPs can be repeated multiple times. It is possible to map a SEQUENCE to a list of Grouped AVPs. This shall be allowed at multiple levels of nesting. Example:

In the translation of InsertSubscriberDataArg to ULA.Subscription-Data, SubscriptionData.CSG-Subscription-Data, which is of type repeated Grouped AVP, maps to ISDArg.csgSubscriptionDataList which is of type list of SEQUENCE

Single valued Enumerated AVP has only value (most likely value 0). Binary Enumerated AVP has two values (0 and 1). In ASN.1 an IE can be of type NULL or of type Boolean. All these are used as flags (to enable or disable something).

Examples:

- In the translation of ISDArg to Subsccription-Data, SubscriptionData.Roaming-Restricted-Due-ToUnsupported-Feature with value 0 is set if ISDArg.roamingRestrictedInSgsnDueTo Unsupported-Feature IE is present.

- Subscription-Data.MDT-User-Consent with values 0 and 1 maps to ISDArg.mdtUserConsent, which is of type Boolean

- Subscription-Data.PS-and-SMS-onlyService-Provision is set to 1 if the parameter ISDArg. PS-and-SMS-onlyService-Provision is present, else 0 is set.

\subsection{Mapping of Routing Parameters}

If an interworking is needed after protocol translation, the routing parameters in the incoming request must be mapped to corresponding routing parameters in the outgoing request.

Diameter and TCAP follow different request routing principles. In the case of Diameter, request routing uses the Destination-Realm AVP, Applic- 
ation ID header field and optionally Destination-Host AVP. In the case of TCAP, request routing uses the Signalling Connection Control Part (SCCP) routing principles. This can be either Point Code (PC)-Subsystem Number (SSN) or Global Title (GT) based routing (refer to [4] for CCITT or [5] for ANSI for the corresponding routing principles). Further, a MAP/CAP operation included in a TCAP component has an Application Context Name (ACN).

Here, Diameter S6a/S6d to MAP Gr will be considered in illustrating a typical request routing scenario during interworking.

In a Diameter S6a/S6d request, the User-Name AVP is a mandatory parameter. The User-Name AVP is comprised of three parts and constitutes the subscriber IMSI:

- 3-digit Mobile Country Code (MCC)

- 2 or 3-digit Mobile National Code (MNC)

- Rest, Mobile Subscriber Identification Number (MSIN)

The combination of the MCC and MNC constitute the Home Realm/Domain or the Destination-Realm of the user.

Optionally, the Destination-Host AVP can be used to identify a specific destination. The Destination-Host AVP may be constructed from a range of digits in the MSIN.

Thus, for a request originating on Diameter S6a/S6d, the PC and/or GT is derived from the User-Name AVP. The mapping of the MCC and MNC to PC and/or GT of the HLR is specified in an IWF routing configuration file, along with other configurable SS7 routing parameters such as family (ANSI or CCITT) and National/International indicator.

The Application ID in the Diameter S6a/S6d request header is mapped onto the destination SSN. The destination SSN value corresponds to GSM MAP HLR (6) in the case. Also, the IWF acts on behalf of the SGSN as seen from the HLR. Thus, the origination SSN value corresponds to GSM MAP SGSN (149) in this case - this is also specified in the IWF configuration file.

The outgoing MAP/CAP operation determined from the Diameter Command-Code [2] is used to compute the $\mathrm{ACN}$ in the outgoing TCAP dialogue.

The Transaction Manager stores the latest origination context for an IMSI. The origination context refers to the MME/SGSN serving the IMSI currently. This is important for a subsequent server-initiated message such as a Cancel Location initiated from an HLR. This is because for a server-initiated mes- 
sage, the destination must be "exactly" identified, which is obtained from the stored origination context.

In case the User-Name AVP is not present, which may be true for Diameter Gy/S13/S13', the Destination-Realm is directly used to map to the corresponding PC and/or GT - for Diameter Gy to CAP Ge, the PC and/or GT resolves to a Prepaid SCP; for Diameter S13/S13' to MAP Gf, the PC and/or GT resolves to an EIR.

In case of Diameter Gy/S13/S13', if the Destination-Host is not present, the destination node may be identified through a configured Load Balancing scheme.

Mapping of MAP Gr to Diameter S6a/S6d can be easily determined now.

\section{Conclusion}

Some aspects of Diameter-CAP interworking has not been discussed in this paper. In the case of Diameter Gy, a single Diameter session can span multiple Diameter transactions. In contrast, in the case of Diameter S6a/S6d, a single Diameter session spans one Diameter transaction.

The Diameter-RADIUS interworking has not been discussed at all in this paper. These two interworking scenarios shall be dealt with in detail in a future version of this paper. ${ }^{1}$

\section{References}

[1] 3GPP TS 29.305: InterWorking Function (IWF) between MAP based and Diameter based interfaces.

[2] IETF RFC 6733: Diameter Base Protocol.

[3] ITU-T Recommendation Q.771: Functional description of transaction capabilities.

[4] ITU-T Recommendation Q.713: Signalling connection control part formats and codes.

[5] ANSI T1.112: Signalling System Number 7 (SS7) ? Signalling Connection Control Part (SCCP).

[6] 3GPP TS 29.002: Mobile Application Part (MAP) specification.

[7] http://www.3gpp.org/ftp/Specs/archive/29_series/29.002/ASN.1/, Cross-reference listing and fully expanded ASN.1 sources of the MAP protocol.

[8] 3GPP TS 29.272: Mobility Management Entity (MME) and Serving GPRS Support Node (SGSN) related interfaces based on Diameter Protocol.

1 The authors of this paper may however be contacted if information on these interworking scenarios is desired. 


\section{Biographies}

Arnab Dey is a Technical Lead at Diametriq, LLC. His interests include architecture and design of software, programming, authoring technical white papers in emerging telecom and web technologies, speaking in various forums and mentoring. Dey received a Bachelors in Electrical Engineering from Jadavpur University, India and an Executive Post Graduate Diploma in Business Management from Symbiosis International University, India. Contact him at adey@diametriq.com.

Balaji Rajappa is a Development Engineering Manager at Diametriq, LLC. His interests include network convergence, cloud computing on telecom networks and rich communication suite. Rajappa received a Bachelors in Engineering from Bharathiyar University, India. Contact him at brajappa@diametriq.com.

Lakshman Bana is a Senior Software Engineer. He has worked on telecommunications signaling protocols for more than 12 years. He is currently focusing on mobile applications development and rich communication services. Bana received an MS in Computer Information Systems from Florida Tech, USA. Contact him at lbana@ diametriq.com. 